\title{
Horizontal impaction of bilateral mandibular second premolars with distal inclination: A case report
}

\author{
Sachiko Hayashi-Sakai*1, Yumiko Maruyama*2 and Yo Taguchi*1 \\ ${ }^{* 1}$ Division of Pediatric Dentistry, Department of Oral Health Science, Course for Oral Life Science, \\ Niigata University Graduate School of Medical and Dental Sciences \\ 2-5274 Gakkocho-dori, Chuo-ku, Niigata 951-8514, JAPAN \\ *2 Department of Nursing, Niigata University Medical and Dental Hospital \\ 1-754 Asahimachi-dori, Chuo-ku, Niigata 951-8520, JAPAN
}

\begin{abstract}
In the Japanese population, the second premolars are the secondmost-frequently impacted teeth in the mandible, after the third molars. Although some papers have reported on the treatment of impacted mandibular second premolars caused by abnormal tilting of the tooth germ, there has been little information regarding bilateral horizontal impaction with distal inclination. The purpose of the present report is to describe the treatment course of a rare and severe case of an 11-year, 8-month-old girl without any obvious etiologies whose bilateral mandibular second premolars were inclined to the distal direction at approximately 90 degree angles, with the root formation developed to an approximately half length. After extraction of the retained primary molars, traction was performed in the treatment of the affected teeth. The affected premolars had fully erupted and achieved occlusion one year after the beginning of intervention. In conclusion, it was considered that the present case could obtain a successful outcome by limited traction since no clinical anomalies or discomfort was detectable during the one year of retention.
\end{abstract}

Key words

Bilateral impaction, Horizontal inclination, Mandibular second premolar

\section{Introduction}

Eruption disturbance involving tooth impaction is defined as a condition in which any tooth fails to erupt into a normal functional position or remains unerupted in the jaw beyond the time at which it should normally erupt ${ }^{1)}$. The condition is caused by several systemic diseases or local etiologic factors. As regards the mandibular premolars, the most frequent causes are space loss and the ectopic position of the tooth germ $^{2-6)}$. Other etiologies are odontomas, supernumerary tooth, ankylosis, and/or an apical lesion of the primary molars ${ }^{7)}$. Some papers have reported on the treatment of impacted mandibular second premolars caused by abnormal inclination of the tooth germ without obvious etiologies ${ }^{6-10)}$.

Received on February 24, 2010

Accepted on April 20, 2010
According to our survey of the literature, however, there has been little information regarding bilateral horizontal impaction with distal inclination of the premolar.

The purpose of the present report is to describe a bilateral impaction of the mandibular second premolars with severe distal inclination in a patient aged 11 years, 8 months, and the treatment course leading to a successful outcome by limited traction.

\section{Case Report}

An 11-year, 8-month-old Japanese girl was referred to the Pediatric Dental Clinic of Niigata University Medical and Dental Hospital from a private dental clinic with a complaint of bilateral impacted mandibular second premolars. The patient had ventricular extrasystole and epilepsy. Although an abnormal electroencephalogram was recognized, the patient 

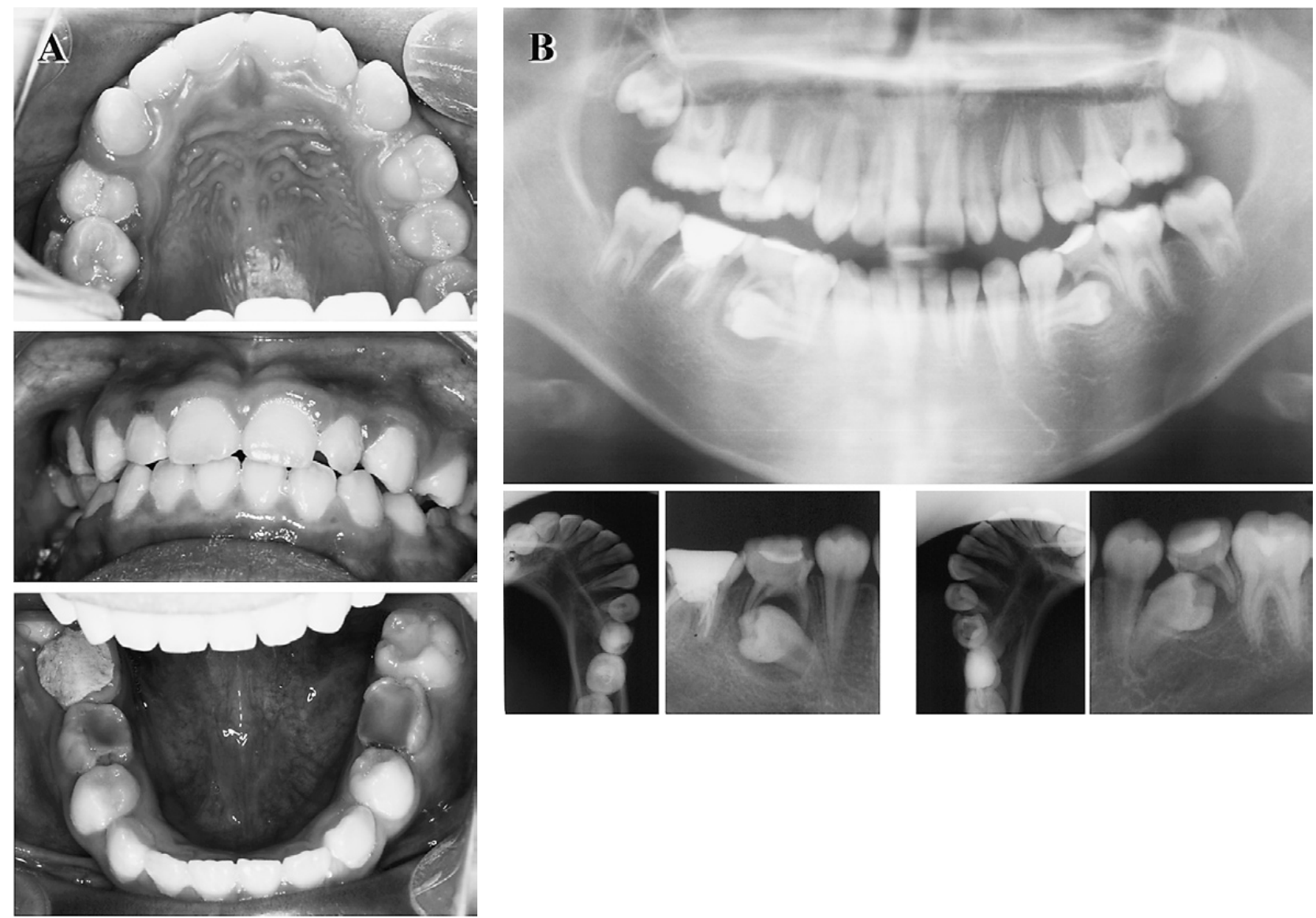

Fig. 1 Clinical intraoral views (A) and radiographic appearance (B) at 11 years, 8 months, before treatment. In B, the top showed orthopantomogram and the bottom showed periapical and occlusal radiographs of the mandibular right and left second premolars. The mandibular second premolars on both sides were impacted horizontally in a distal direction.

was well followed by a pediatrician and had no limits in her daily life. There was no relevant family history of medical or dental abnormalities.

The orthopantomogram revealed that mandibular second premolars on both sides were impacted horizontally in a distal direction, and that the predecessors had received no root canal treatment by the referring dentist and showed prolonged retention (Fig. 1). The root of each impacted premolar was formed to approximately half length.

One month after extraction of the second primary molars, fenestration and traction were performed on the mandibular right second premolar. For the mandibular left second premolar, only traction was needed without fenestration because part of the affected tooth appeared spontaneously in the oral cavity. The appliance for traction was composed of two parts, a sectional arch in the buccal side and a lingual arch with a hook for traction (Fig. 2). The traction of the second premolars was performed by orthodontic elastics toward the mesial direction from buccal and lingual sides, and the elastics were changed once a month. The non-activated coils were inserted into the sectional arch to prevent distal inclining of the first premolars and to keep the eruption spaces for the impacted teeth. The patient was told that if this treatment failed to mobilize the affected teeth, prosthetic management would be indicated. Due to the advanced root development, surgical repositioning and autotransplantation would not be viable treatment alternatives ${ }^{6}$.

Three months after traction, the occlusal surface of the mandibular right second premolar could be seen in the oral cavity, but was inclined to the distal direction. The mandibular left second premolar remained tilted approximately 90 degrees toward the distal direction (Fig. 3). The appliance was adjusted to up-right the premolars toward the mesial direction 

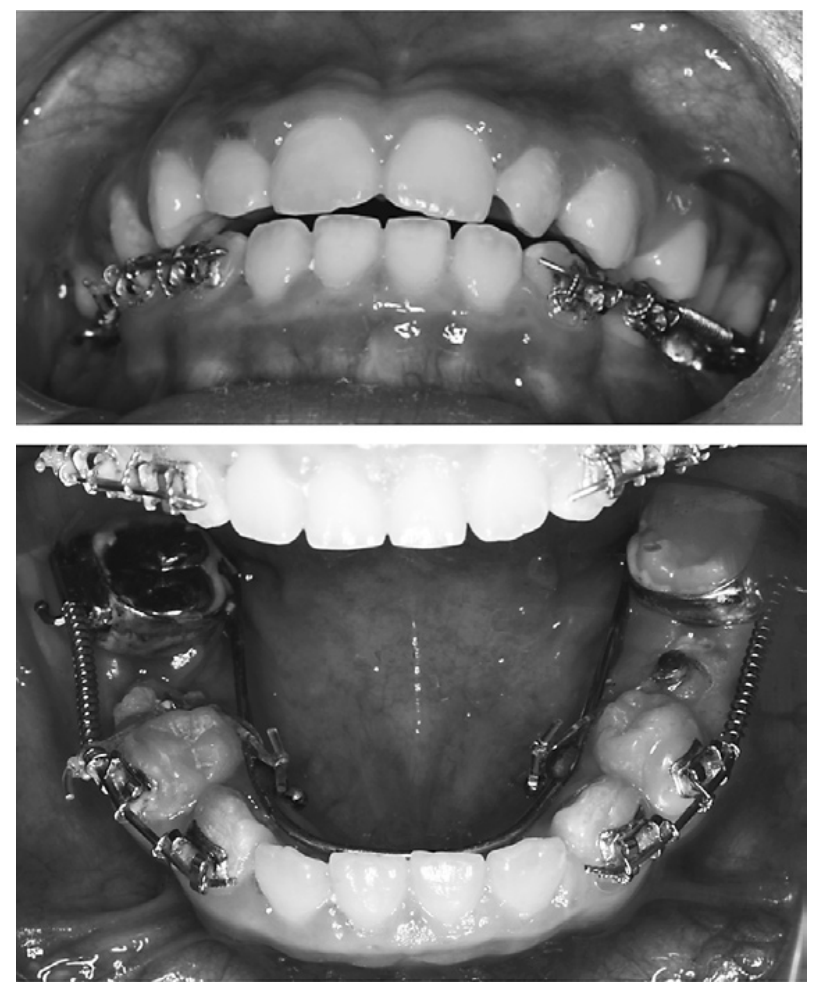

Fig. 2 The appliance for traction composed of two parts, a sectional arch in the buccal side and a lingual arch with a hook for traction. The traction of the second premolars was performed by orthodontic elastics toward the mesial direction from buccal and lingual sides, and the elastics were changed once a month. The non-activated coils were inserted into the sectional arch to prevent distal inclining of the first premolars and to keep the eruption spaces for the impacted teeth.

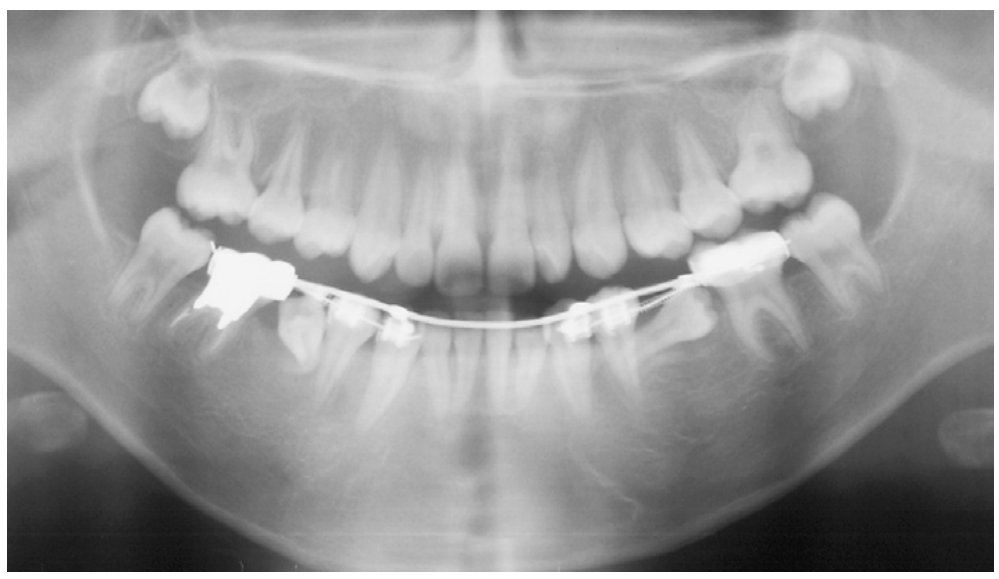

Fig. 3 Orthopantomogram at 12 years, 1 month. The mandibular right second premolar was inclined to the distal direction. The mandibular left second premolar remained tilted approximately 90 degrees toward the distal direction.

and to align them within the dental arch.

Six months after traction, the appliance was changed to an orthodontic full bracket type, and adjustments were performed once a month. As a result, both mandibular second premolars were aligned and successfully achieved occlusion one year after the beginning of intervention, though the root apex formations of the mandibular second 

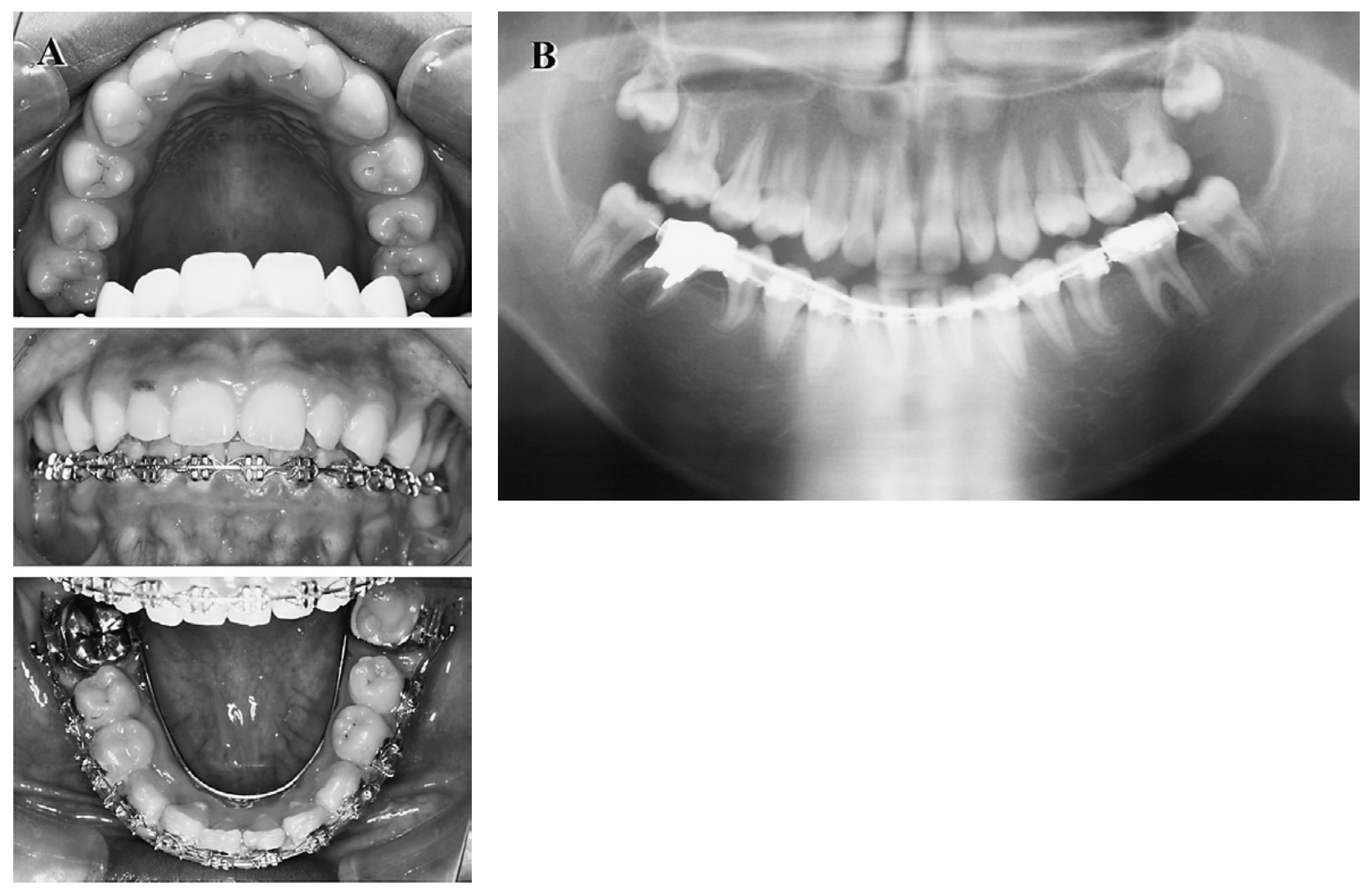

Fig. 4 Clinical intraoral views (A) and orthopantomogram (B) at 12 years, 11 months, after treatment. Both mandibular second premolars were aligned and successfully achieved occlusion one year after the beginning of intervention, though the root apex formations of the mandibular second premolars were slightly dilacerated.

premolars were slightly dilacerated (Fig. 4). To date, a retainer has been used for a period of one year.

\section{Discussion}

Pediatric dentists often encounter the problem of tooth impaction. The mandibular second premolars are fourth-most-commonly impacted in Japanese children, after the maxillary central incisors, canines, and the first molar in Japanese children ${ }^{11)}$. The overall frequency of mandibular premolar impaction in all populations has been found to range from 0.1 to $0.3 \%{ }^{8,12,13)}$. According to our recent study on eruption disturbance, mandibular premolars accounted for $7.6 \%$ of all eruption disturbances in our clinic ${ }^{11}$. Except for the obvious etiologies such as space loss, ectopic position of the tooth germ, odontomas, supernumerary teeth, and apical lesions in the primary molars, there are few reports regarding mandibular premolar impactions caused by abnormal inclination of the tooth germ $^{6-8,13)}$. In the present case, it was speculated that the mandibular premolar impaction was caused by idiopathic abnormal inclination of the tooth germ, since the predecessors had never received root canal treatment and there was no obvious local factor.

As to the treatment of distally inclined mandibular premolars, Andreasen ${ }^{8)}$ has recommended extraction of the primary molar to activate and guide the eruption process when the primary molar and the permanent premolar are in close proximity. He has also proposed that surgical exposure is effective in cases where the axial tilt of the premolar is limited to 45 degrees, whereas exposure in cases where the axial tilt comes close to 90 degrees gives unpredictable results. Takagi et al. found in 10 distally inclined cases a positive correlation between the degree of distal inclination and the delay in formation of the premolar tooth germ $^{10)}$. They have recommended extraction of the predecessor and bone fenestration of the affected premolar at the developing stage of initiation or one-fourth of the tooth root formation, 
often followed by traction, when the mandibular second premolar showed aberrant tilting impaction of more than 65 degrees. Since the present impacted mandibular second premolars were inclined to the distal direction at approximately 90 degrees and their roots were already formed up to approximately half length, it was decided that traction should be needed immediately after extraction of the predecessors. However, Collett ${ }^{6)}$ has described a case in which only removal of a deciduous second molar was followed by eruption of a horizontally inclined premolar located deep within the alveolus, and the premolar could erupt to the level of the occlusal plane without fenestration and traction 16 months after removal of the predecessor. It is suggested that further case accumulation can help in determining the treatment plan for severely inclined premolars.

It has been reported that the degree of distal inclination is statistically greater in premolars with unilateral than with bilateral impaction, and that traction was seldom needed in the bilateral cases ${ }^{10)}$. Clearly, the conditions and treatment guidelines of this previous study did not apply to the present case. It is therefore necessary to further investigate and compare unilaterally and bilaterally impacted mandibular second premolars.

\section{References}

1) Pindborg, J.J.: Impaction of teeth. In: Pathology of the Dental Hard Tissues. Munksgaard, Copenhargen, 1970, pp. 241-247.

2) Johnsen, D.C.: Prevalence of delayed emergence of permanent teeth as a result of local factors. JADA 94: 100-106, 1997.

3) Siervo, S., Pampalone, A., Siervo, P., Cerri, E., Bandettini, B. and Siervo, R.: Rescue of "hopeless" second premolar. Oral Surg Oral Med Oral Pathol 76: 276-278, 1993.

4) Shapira, Y., Borell, G., Kuftinec, M.M. and Nahlieli, O.: Bringing impacted mandibular second premolars into occlusion. JADA 127: 1075-1078, 1996.

5) Kokichi, V.G. and Mathews, D.P.: Surgical orthodontic management of impacted teeth. JNJ Dent Assoc 70: 42-45, 1999.

6) Collett, A.R.: Conservative management of lower second premolar impaction. Aust Dent J 45: 279-281, 2000.

7) Kobaiashi, V.T., Mitomi, T., Taguchi, Y. and Noda, T.: Occlusal guidance for eruption disturbance of mandibular second premolar: a report of three cases. J Clin Pediatr Dent 27: 101-105, 2003.

8) Andreasen, J.O.: The impacted premolar. In: Textbook and Color Atlas of Tooth Impaction. Munksgaard, Copenhargen, 1997, pp. 177-195.

9) Murray, P. and Brown, N.L.: The conservative approach to managing unerupted lower premolartwo case reports. Int J Paediatr Dent 13: 198-203, 2003.

10) Takagi, J., Sano, T., Taguchi, Y. and Mitomi, T.: A clinical study of eruption disturbance of the mandibular second premolar by the ectopic position of the tooth germ. Jpn J Ped Dent 47: 433-441, 2009. (in Japanese)

11) Noda, T., Takagi, M., Hayashi-Sakai, S. and Taguchi, Y.: Eruption disturbances in Japanese children and adolescents. Ped Dent J 16: 50-56, 2006.

12) Grover, P.S. and Lorton, L.: The incidence of unerupted permanent teeth and related clinical cases. Oral Surg Oral Med Pathol 59: 420-425, 1985.

13) Kramer, R.M. and Williams, A.C.: The incidence of impacted teeth. Oral Surg 29: 237-241, 1970. 